\title{
Patients with polyclonal hepatocellular carcinoma are at a high risk of early recurrence and have a poor recurrence-free survival period
}

\author{
Masaki Kaibori ${ }^{1} \cdot$ Kazuko Sakai $^{2} \cdot$ Hideyuki Matsushima $^{1} \cdot$ Hisashi Kosaka ${ }^{1} \cdot$ Kosuke Matsui $^{1} \cdot$ Marco A. De Velasco $^{2}$. \\ Mitsugu Sekimoto ${ }^{1} \cdot$ Kazuto Nishio $^{2}$ (1)
}

Received: 4 August 2021 / Accepted: 12 November 2021 / Published online: 1 January 2022

(c) The Author(s) 2021

\begin{abstract}
Background/purpose of the study Tumor heterogeneity based on copy number variations is associated with the evolution of cancer and its clinical grade. Clonal composition (CC) represents the number of clones based on the distribution of B-allele frequency (BAF) obtained from a genome-wide single nucleotide polymorphism (SNP) array. A higher CC number represents a high degree of heterogeneity. We hypothesized and evaluated that the CC number in hepatocellular carcinoma (HCC) tissues might be associated with the clinical outcomes of patients.

Methods Somatic mutation, whole transcriptome, and CC number based on copy number variations of 36 frozen tissue samples of operably resected HCC tissues were analyzed by targeted deep sequencing, transcriptome analysis, and SNP array. Results The samples were classified into the heterogeneous tumors as poly-CC $(n=26)$ and the homogeneous tumors as mono-CC $(n=8)$. The patients with poly-CC had a higher rate of early recurrence and a significantly shorter recurrencefree survival period than the mono-CC patients ( 7.0 months vs. not reached, $p=0.0084)$. No differences in pathogenic nonsynonymous mutations, such as TP53, were observed between the two groups when targeted deep sequencing was applied. A transcriptome analysis showed that cell cycle-related pathways were enriched in the poly-CC tumors, compared to the mono-CC tumors. Poly-CC HCC is highly proliferative and has a high risk of early recurrence.

Conclusion $\mathrm{CC}$ is a possible candidate biomarker for predicting the risk of early postoperative recurrence and warrants further investigation.
\end{abstract}

Keywords Clonal composition · Hepatocellular carcinoma $\cdot$ Recurrence-free survival $\cdot$ Copy number variation $\cdot$ Gene expression $\cdot$ Gene set enrichment analysis $\cdot$ Early recurrence $\cdot$ Single nucleotide polymorphism array $\cdot$ B-allele frequency Proliferation

\section{Abbreviations}

AFP Alpha-fetoprotein

ALT Alanine aminotransferase

BAF B-allele frequency

$\mathrm{CC} \quad$ Clonal composition

$\mathrm{CCP} \quad$ Comprehensive cancer panel

Masaki Kaibori and Kazuko Sakai contributed equally to this work.

Kazuto Nishio

knishio@med.kindai.ac.jp

1 Department of Surgery, Hirakata Hospital, Kansai Medical University, Hirakata, Osaka, Japan

2 Department of Genome Biology, Faculty of Medicine, Kindai University, 377-2 Ohno-Higashi, Osaka-Sayama, Osaka 589-8511, Japan
FATHMM Functional analysis through hidden Markov models

FC Fold change

FDR False discovery rate

FFPE Formalin-fixed, paraffin-embedded

GO Gene ontology

GSEA Gene set enrichment analysis

HBV Hepatitis B virus

HCC Hepatocellular carcinoma

$\mathrm{HCV} \quad$ Hepatitis $\mathrm{C}$ virus

ICGR15 Indocyanine green retention rate at $15 \mathrm{~min}$

IFN $\gamma \quad$ Interferon gamma

IGV Integrative genomics viewer

IL6 Interleukin-6

JAK Janus-associated kinase

MIP Molecular inversion prove

MOMA Number of minor alleles 


\begin{tabular}{|c|c|}
\hline MSigDB & Molecular signatures database \\
\hline NBNC & Non-hepatitis B and non-hepatitis C virus \\
\hline NFкB & Nuclear factor-kappa B \\
\hline OS & Overall survival \\
\hline PIVKA-II & $\begin{array}{l}\text { Protein induced by vitamin } \mathrm{K} \text { absence or } \\
\text { antagonist-II }\end{array}$ \\
\hline PTPE & $\begin{array}{l}\text { Percutaneous transhepatic portal vein } \\
\text { embolization }\end{array}$ \\
\hline RFA & Radiofrequency ablation \\
\hline RFS & Recurrence-free survival \\
\hline STAT3 & $\begin{array}{l}\text { Signal transducer and activator of transcrip } \\
\text { tion } 3\end{array}$ \\
\hline TACE & Transcatheter arterial chemoembolization \\
\hline TMB & Tumor mutation burden \\
\hline TNF $\alpha$ & Tumor necrosis factor alpha \\
\hline
\end{tabular}

\section{Introduction}

Liver cancer is one of the most common malignancies, with more than 800,000 new cases diagnosed globally each year [1]. Hepatocellular carcinoma (HCC) accounts for approximately $75 \%$ of liver cancers. Although surgical resection improves the survival of HCC patients, early recurrence after hepatic resection is a poor prognostic factor for patients with HCC. HCC is a highly heterogeneous cancer [2-4]. Molecular heterogeneity and a lack of biomarkers for recurrence have contributed to the poor prognosis of HCC patients.

The intratumoral heterogeneity of cancer cells remains largely unexplored. Copy number variations reflect genomic structural changes that give rise to gene amplification or deletion. Chromosomal microarray and other array-based approaches have been widely adopted for the detection of whole-genome copy number variations $[5,6]$. The OncoScan FFPE Assay Kit (Thermo Fisher Scientific, Wilmington, DE) relies on molecular inversion prove (MIP) technology to detect genome-wide copy number alterations, loss of heterozygosity, and somatic mutation [7]. This assay provides the B-allele frequency (BAF), the $\log 2$ ratio $(\log 2 \mathrm{R})$, and the copy number for each of over 220,000 analyzed polymorphic genomic locations. The copy number is derived from the $\log 2 \mathrm{R}$ and $\mathrm{BAF}$ values. The clonal composition of a tumor can be analyzed based on the BAF and $\log 2 \mathrm{R}$, which can be determined from the whole-genome copy number profiles obtained using the OncoScan FFPE Assay Kit. We previously demonstrated that the whole-genome single nucleotide polymorphism (SNP) array could be applied to the detection of the clonal composition of human ovarian cancer. Genome-wide segmentation data consisting of the $\log 2 \mathrm{R}$ and $\mathrm{BAF}$ have been previously used to estimate the clonal composition (CC) number for individual tumors [8]. A similar approach resulted in a report indicating that the clonal composition can be derived from both somatic mutations as well as the $\log 2 \mathrm{R}$ and BAF for loci with an aberrant copy number [9]. The $\mathrm{CC}$ number is not equivalent to the number of clones in the tumor tissue, but instead represents and estimates the number of clones that show copy number changes. For instance, a BAF value of 0.5 indicates a heterozygous genotype $(\mathrm{AB})$, whereas values of 0 and 1 indicate homozygous genotypes (AA and $\mathrm{BB}$, respectively). The BAF distribution in segments with copy number changes suggests the presence of different clones with different BAF patterns intratumorally. In segments with copy number gains, the percentage of aberrant cells with different BAF patterns due to BAF variation is difficult to evaluate because the copy number gain is not constant. On the other hand, there is no such limitation in evaluating the BAF in segments with copy number loss, and the clonal composition number can be derived from the presence of aberrant cells with a different BAF pattern.

We hypothesized that HCC with a high CC would exhibit highly aggressive behaviors when compared with $\mathrm{HCC}$ with a low CC. We examined the CC numbers of previously collected frozen tissue samples of HCC and investigated the association with early recurrence in postoperative HCC patients. In addition, we also used NGS-based deep sequencing to detect somatic mutations and to examine the whole transcriptome of HCC tissues to investigate any associations with CC.

\section{Materials and methods}

\section{Hepatocellular carcinoma samples}

Frozen tumor tissue samples were obtained from 36 patients who underwent surgical resection at Kansai Medical University Hospital between August 2011 and December 2013. All the patients provided written informed consent to participate in the study, including the collection of tumor frozen tissues for analysis. Tumor stage is defined by General Rules for the Clinical and Pathological Study of Primary Liver Cancer [10] as stage I-IVa. All cases including five cases of IVa tumors were completely resected. The early recurrence of postoperative HCC has been previously defined as recurrence within 1 year of resection $[11,12]$. The recurrence-free survival (RFS) and overall survival (OS) were defined as the time interval between the date of hepatectomy and the date of recurrence or death, respectively. After surgery, patients were followed in the outpatient clinic every 3 months for the first 2 years and every 6 months thereafter. Early recurrence was defined as the appearance of a new lesion compatible with HCC on radiologic examination during the follow-up period. 


\section{Isolation of genomic DNA and RNA}

A $0.5 \mathrm{~cm}$ square tumor legion without necrosis visually was sectioned and used for the analysis. DNA and RNA were isolated from frozen tissues using an AllPrep DNA/RNA Mini Kit (Qiagen, Valencia, CA) according to the manufacturer's instructions. The quality and quantity of the nucleic acid were verified using a NanoDrop 2000 device, PicoGreen dsDNA Reagent, and RiboGreen RNA reagent (all from Thermo Scientific).

\section{Microarray-based comparative genomic hybridization assay}

A MIPs array for the genome-wide estimation of copy number aberrations was performed using the OncoScan FFPE Assay Kit (Thermo Fisher Scientific), as previously reported [8]. Briefly, $80 \mathrm{ng}$ of DNA was subjected to annealing with these MIPs for 16-18 $\mathrm{h}$, followed by enzyme digestion and two separate gap-fill reactions. The circular MIPs were then separately linearized for each gap fill with a cleavage enzyme and amplified using PCR. The PCR products were subjected to enzymatic cleavage and fragmentation, followed by hybridization for 16-18 h with two OncoScan arrays (one for each gap fill). The arrays were then stained and washed using a GeneChip Fluidics Station 450 and loaded into a GeneChip Scanner 3000 7G (Affymetrix). Array fluorescence intensity (CEL) files were generated using Affymetrix GeneChip Command Console (AGCC) software, version 4.0, and the CEL files were converted to OSCHP files using OncoScan Console software 1.3 and visualized with Chromosome Analyses Suite software (version 4.0). A representative whole-genome view is shown in Fig. S1.

\section{Clonal composition analysis}

Clonal composition numbers were calculated using BAF and $\log 2 \mathrm{R}$ information obtained from the OncoScan FFPE Assay using the Onco Clone Composition program, as described previously [8]. A given copy number segment can be associated with a percentage of aberrant tumor cells $(\% \mathrm{AC})$ and is assumed to result from a single underlying event attributable to a single clone; in other words, the copy number segments are associated with the same \% AC and belong to the same clone. The clonal composition number is calculated by identifying the number of different \% AC detected among the aberrant segments. The number of minor alleles (NOMA) at a heterozygous site can be defined as the BAF of a segment. The calculation of clone composition number only uses NOMA $=0$, which represents a segment with $100 \%$ loss of heterozygosity and a normal copy number. If only one of such alleles is present, the BAF for such a segment will be zero. The Onco Clone Composition program provides analytical estimations of clonal composition using a clustering approach that clusters all segments with the same $\log \mathrm{R}$ and BAF and then combines clusters corresponding to the same \%AC. A graphical representation of the aberrated segments and their association with \% AC is shown in Supplementary Fig. S2.

\section{Targeted DNA sequencing}

A targeted DNA library comprising approximately $1.2-\mathrm{Mb}$ of the coding regions of 409 genes for panel sequencing was constructed using an Ion AmpliSeq Comprehensive Cancer Panel (CCP) (Thermo Fisher Scientific), as described previously [13]. Briefly, $40 \mathrm{ng}$ of DNA was subjected to multiplex PCR amplification using an Ion AmpliSeq Library Kit 2.0 and the Ion AmpliSeq Comprehensive Cancer Panel (Thermo Fisher Scientific), which covers all exons in 409 genes. After multiplex PCR, Ion Xpress Barcode Adapters (Thermo Fisher Scientific) were ligated to the PCR products, which were then purified using Agencourt AMPure XP beads (Beckman Coulter, Brea, CA). The purified libraries were pooled and then sequenced using an Ion Torrent S5 instrument and the Ion 550 Chip Kit (all from Thermo Fisher Scientific). DNA sequencing data were accessed through the Torrent Suite ver. 5.10 program (Thermo Fisher Scientific). Reads were aligned against the hg19 human reference genome, and variants were called using Variant Caller, ver. 5.10. Raw variant calls were filtered with a quality score of $<100$ and were manually checked using the integrative genomics viewer (IGV; Broad Institute, Cambridge, MA). Germline mutations were excluded using the Human Genetic Variation Database (http://www.genome.med.kyoto-u.ac.jp/ SnpDB) and the Exome Aggregation Consortium database. Variants with FATHMM scores greater than 0.7 were classified as pathogenic [14]. The tumor mutation burden (TMB mutations/Mb) was assessed using Ion Reporter Software, ver. 5.10 (Thermo Fisher Scientific).

\section{Whole transcriptome analysis}

The whole transcriptome analysis was performed using the AmpliSeq Transcriptome Human Gene Expression Kit (Thermo Fisher Scientific). For library preparation, cDNA was generated using the SuperScript VILO cDNA Synthesis kit (Thermo Fisher Scientific) from $10 \mathrm{ng}$ of total RNA. Then, cDNA was amplified for 12 cycles by adding PCR Master Mix and the AmpliSeq human transcriptome gene expression primer pool (Thermo Fisher Scientific). After multiplex PCR, Ion Xpress Barcode Adapters (Thermo Fisher Scientific) were ligated to the PCR products, which were then purified using Agencourt AMPure XP beads (Beckman Coulter). The purified libraries were pooled and then sequenced using an Ion Torrent S5 instrument and the 
Ion 550 Chip Kit (all from Thermo Fisher Scientific). The Ion Torrent Suite v5.10 software (Thermo Fisher Scientific) was used for base calling, alignment to the human reference genome (hg19), and quality control. Raw reads were then analyzed automatically using the AmpliSeqRNA plugin to generate gene-level expression values for all 20,802 RefSeq human genes.

\section{Gene set enrichment analysis (GSEA)}

A gene-set enrichment analysis (GSEA) was performed to identify pathways enriched in the Molecular Signatures Database (MSigDB) Hallmark gene set [15, 16]. A nominal $p$ value of $<0.05$ and an FDR (false discovery rate) $q$ value of $<0.05$ were considered statistically significant.

\section{Gene selection and pathway analysis}

A total of 2408 genes that were differentially expressed according to $\mathrm{CC}$ numbers were selected using the following criteria: $p$ value $<0.05$ and FDR $q$ value $<0.5$. Among these 2408 genes, the top 500 markers of differential expression were identified based on the signal-to-noise ratio. Hierarchical clustering was performed using one minus the Pearson correlation coefficient as a distance measure and the average linkage method. To explore the potential biological pathways, genes in a cluster were submitted to the Metascape tool (https://metascape.org/gp/index.html) [17].

\section{Tumor infiltration analysis}

Tumor IMmune Estimation Resource (TIMER2.0, http:// timer.comp-genomics.org/) was used to estimate immune infiltration levels of immune cells including B cell, CD4+ T cell, CD8+ T cell, Neutrophil, Macrophage, and Myeloid dendritic cell [18-20]. The gene expression data from whole transcriptome analysis was normalized as RPM (reads per million mapped reads).

\section{Immunohistochemical staining}

A standard immunohistochemistry analysis was performed for the cytokeratin 19 (CK19) and Epithelial cell adhesion molecule (EpCAM) as follows. Formalin-fixed tissue sections were sectioned and placed on positively charged slides and pretreated with steam heating in DAKO target retrieval solution (Catalog \#S1699, Agilent, Santa Clara, CA) for $20 \mathrm{~min}$. Slides were incubated with anti-CK19 antibody (Catalog \#13092, Cell Signaling, Danvers, MA, dilution 1:100) and anti-EpCAM antibody (Catalog \#14452, Cell Signaling, dilution 1:100), overnight stained using the $\mathrm{ABC}$ kit (Vector Laboratories, Burlingame, CA) following the manufacturer's protocols, developed in diaminobenzidine
DAB (Invitrogen, Carlsbad, CA), and counterstained with hematoxylin. Assessment of immunohistochemical staining was performed on scanned colon mucosa sections captured at $10 \times$ magnification. $\mathrm{H}$ scores were calculated for CK19 or EpCAM positivity in the cancerous regions using QuPath v0.3.0-m4 image analysis software (https://qupath.github. io/).

\section{Statistical analysis}

Categorical variables were compared using the Fisher exact test. Continuous variables were compared between groups with the Mann-Whitney $U$ test. Variables with a statistical significance in the univariate analysis were included in the multiple logistic regression analysis. Survival was estimated using the Kaplan-Meier method and the log-rank test method. All the statistical analyses were performed using JMP software, version 14.2 (SAS Institute, Cary, NC), and Prism software, version 8.4 (GraphPad Software, San Diego, CA). A $p$ value of $<0.05$ was considered statistically significant.

\section{Results}

\section{Clinicopathological characteristics of patients}

Clinicopathological characteristics of patients are shown in Table 1. The median age was 71.5 years (range 50-87), and 22 patients $(61.1 \%)$ were male gender. Thirty-three patients (91.7\%) were Child-Pugh class A. Patients with HBV and HCV infection were 7 (19.4\%) and 14 (38.9\%), respectively. The median ALT level was 29.5 U/l (range 9-92), total bilirubin level was $0.7 \mathrm{mg} / \mathrm{dl}$ (range 0.3-1.6), the median albumin level was $4.15 \mathrm{~g} / \mathrm{dl}$ (range 2.6-5), median AFP level was $37.6 \mathrm{ng} / \mathrm{ml}$ (range 2-261,351), and the median PIVKAII level was $93 \mathrm{mAU} / \mathrm{ml}$ (range 13-75,000). The median ICGR15 value was $12.9 \%$ (range 2.3-50.1). Seven patients received preoperative treatment included monotherapy of transcatheter arterial chemoembolization (TACE, $n=5$ ), combination therapy of TACE and percutaneous transhepatic portal vein embolization (PTPE, $n=1$ ), combination therapy of TACE and radiofrequency ablation (RFA, $n=1)$. All patients with stage I-IVa received curable resection, and the maximum tumor diameter was $4 \mathrm{~cm}$ (range 0.8-140). The number of tumors ranged from 1 to 3 . Patients classified into well $(8 / 36,22.2 \%)$, moderately $(24 / 36,66.7 \%)$, and poor (2/36, 5.6\%) differentiated HCC. Microscopic vascular invasion was noted in $83.3 \%$ (30/36) of patients. Early recurrence within 12 months after curative resection occurred in 22 patients $(61.1 \%)$. All patients with recurrence had intrahepatic recurrence. Distant metastases were found in the lung (1 patient) and left adrenal gland (1 patient). 
Table 1 Patients characteristics

\begin{tabular}{|c|c|c|}
\hline Variable & Category & $n=36$ \\
\hline Sex & Male/Female & 22/14 (61.1/38.9) \\
\hline Age & Median (range) & $71.5(50-87)$ \\
\hline Liver function status & Child-Pugh A/B & $33 / 3(91.7 / 8.3)$ \\
\hline Etiology & HBV/HCV/alcoholic/normal & $7 / 14 / 2 / 13(19.4 / 38.9 / 5.6 / 36.1)$ \\
\hline $\operatorname{ALT}(\mathrm{U} / \mathrm{l})$ & Median (range) & $29.5(9-92)$ \\
\hline Total bilirubin (mg/dl) & Median (range) & $0.7(0.3-1.6)$ \\
\hline Albumin (g/dl) & Median (range) & $4.15(2.6-5)$ \\
\hline ICGR15 (\%) & Median (range) & $12.9(2.3-50.1)$ \\
\hline $\operatorname{AFP}(\mathrm{ng} / \mathrm{ml})^{\mathrm{a}}$ & Median (range) & $37.6(2-261,351)$ \\
\hline PIVKA-II (mAU/ml $)^{\mathrm{a}}$ & Median (range) & $93(13-75,000)$ \\
\hline Tumor size $(\mathrm{cm})^{\mathrm{a}}$ & Median (range) & $4(0.8-140)$ \\
\hline Tumor number & Median (range) & $1(1-3)$ \\
\hline Microscopic vascular invasion & Positive/negative & $30 / 6(83.3 / 16.7)$ \\
\hline Histologic grade & well/moderate/poor/unknown & $8 / 24 / 2 / 2(22.2 / 66.7 / 5.6 / 5.6)$ \\
\hline Stage $^{a}$ & $\mathrm{I} / \mathrm{II} / \mathrm{III} / \mathrm{IVa}$ & $1 / 8 / 21 / 5(2.9 / 22.9 / 60.0 / 14.3)$ \\
\hline History of previous treatment & Present/absent & $7 / 29(19.4 / 80.6)$ \\
\hline Early recurrence & Yes/no & $22 / 14(61.1 / 38.9)$ \\
\hline
\end{tabular}

The asterisk indicates statistical significance

${ }^{\mathrm{a}}$ Missing data in one patient

\section{Clonal composition number of resectable hepatocellular cancer}

We analyzed 36 frozen samples of HCC using a wholegenome SNP array (Fig. S1) and estimated the clonal composition number using the Onco Clone Composition estimation program (Fig. S2). The mean $\pm \mathrm{SD}$ value for the $\mathrm{CC}$ number was $1.0 \pm 0.8$ (range $0-3$ ). It should be noted that a case with a $\mathrm{CC}$ number of 0 indicates a homogenous tumor mass. The $\mathrm{CC}$ number could not be estimated in two cases because the copy number data did not plot onto the fitting curve in the program. Representative plots for the CC profile are shown in Fig. S2. The HCC samples were categorized into mono- $\mathrm{CC}(\mathrm{CC}=0, n=8)$ and poly- $\mathrm{CC}(\mathrm{CC} \geq 1, n=26)$ groups that respectively reflected homogenous and heterogenous clones.

\section{Clinicopathological features associated with clonal composition status}

The clinicopathological characteristics of patients with mono-CC or poly-CC are summarized in Table 2. No significant differences were observed in sex, mean age, liver function status (Child-Pugh A or B), or stage as determined using the Fisher exact test. The presence of microscopic vascular invasion $(p=0.0180)$, moderately to poorly differentiated HCC $(p=0.0469)$, absence of neoadjuvant therapy $(p=0.0374)$, the presence of single tumor nodule $(p=0.0374)$, and higher levels of PIVKA-II $(p=0.0351)$ were significantly frequent in poly-CC group. This result suggests that poly-CC shows an aggressive tumor phenotype compared to mono-CC group. Early recurrence was significantly frequent in the poly-CC group than in the mono-CC group $(p=0.0127)$. There was no difference in the incidence of extrahepatic metastasis between poly-CC and mono-CC. The RFS and OS of the mono- and poly-CC groups were compared (Fig. 1). The median RFS was not reached in the mono-CC HCC groups and 7.0 months in the poly-CC HCC groups. The RFS of patients with poly-CC HCC was significantly shorter than that of the mono-CC patients (Fig. 1a, p=0.0084). The OS of patients with poly-CC tended to be shorter than that of patients with mono-CC, although the difference was not significant (Fig. 1b, $\mathrm{p}=0.2966$ ). Since poly-CC status appeared to be associated with the poorly differentiated HCC, we investigated the relationship with progenitor markers, cytokeratin 19 (CK19) and epithelial cell adhesion molecule (EpCAM). No significant difference of CK19 expressions was observed between mono- and poly$\mathrm{CC}$ groups determined by immunohistochemistry. On the other hand, higher expression of EpCAM was observed in mono-CC than poly-CC significantly $(p=0.0304)$. Thus, $\mathrm{CC}$ status may not indicate the features of progenitor cells (Fig. S3). 
Table 2 Clinicopathological features associated with clonal composition in surgical resected hepatocellular carcinoma

\begin{tabular}{|c|c|c|c|c|}
\hline Variable & Category & $\mathrm{CC}=0(n=8)$ & $\mathrm{CC} \geq 1(n=26)$ & $p$ \\
\hline Sex & Male/female & $6 / 2(75.0 / 25.0)$ & $15 / 11(57.7 / 42.3)$ & 0.4438 \\
\hline Age & $<70 / \geq 70$ & $3 / 5(37.5 / 62.5)$ & $11 / 15(42.3 / 57.7)$ & 1.0000 \\
\hline Liver function status & Child-Pugh A/B & $7 / 1(87.5 / 12.5)$ & $24 / 2(92.3 / 7.7)$ & 1.0000 \\
\hline Etiology & HBV/HCV/alcoholic/normal & $1 / 5 / 0 / 2(12.5 / 62.5 / 0 / 25.0)$ & $5 / 8 / 2 / 11(19.2 / 30.8 / 7.7 / 42.3)$ & 0.5036 \\
\hline $\operatorname{ALT}(\mathrm{U} / \mathrm{l})$ & $<30 / \geq 30$ & $2 / 6(25.0 / 75.0)$ & $14 / 12(53.8 / 46.2)$ & 0.2327 \\
\hline Total bilirubin (mg/dl) & $<1 / \geq 1$ & $6 / 2(75.0 / 25.0)$ & $18 / 8(69.2 / 30.8)$ & 1.0000 \\
\hline Albumin (g/dl) & $<4 / \geq 4$ & $3 / 5(37.5 / 62.5)$ & $11 / 15(42.3 / 57.7)$ & 1.0000 \\
\hline ICGR15 (\%) & $<15 / \geq 15$ & $5 / 3(62.5 / 37.5)$ & $15 / 11(57.7 / 42.3)$ & 1.0000 \\
\hline $\operatorname{AFP}(\mathrm{ng} / \mathrm{ml})^{\mathrm{a}}$ & $<20 / \geq 20$ & $4 / 4(50.0 / 50.0)$ & $10 / 15(40.0 / 60.0)$ & 0.6951 \\
\hline PIVKA-II (mAU/ml $)^{\mathrm{a}}$ & $<40 / \geq 40$ & $6 / 2(75.0 / 25.0)$ & $7 / 18(28.0 / 72.0)$ & $0.0351 *$ \\
\hline Tumor size $(\mathrm{cm})$ & $<4.0 / \geq 4.0$ & $5 / 3(62.5 / 37.5)$ & $11 / 15(42.3 / 57.7)$ & 0.4290 \\
\hline Tumor number & Single/multiple & $4 / 4(50.0 / 50.0)$ & $23 / 3(88.5 / 11.5)$ & $0.0374 *$ \\
\hline Microscopic vascular invasion & Positive/negative & $4 / 4(50.0 / 50.0)$ & $24 / 2(92.3 / 7.7)$ & $0.0180^{*}$ \\
\hline Histologic grade ${ }^{\mathrm{b}}$ & Well/moderate, poor & $4 / 3(57.1 / 42.9)$ & $4 / 21(16.0 / 84.0)$ & $0.0469 *$ \\
\hline Stage $^{c}$ & I/II/III/IVa & $1 / 2 / 2 / 2(14.3 / 28.6 / 28.6 / 28.6)$ & 0/5/18/3 (0/19.2/69.2/11.5) & 0.0819 \\
\hline History of previous treatment & Present/absent & $4 / 4(50.0 / 50.0)$ & $3 / 23(11.5 / 88.5)$ & $0.0374 *$ \\
\hline Early recurrence & Yes/no & $2 / 6(25.0 / 75.0)$ & $20 / 6(76.9 / 23.1)$ & $0.0127 *$ \\
\hline
\end{tabular}

The asterisk indicates statistical significance

${ }^{\mathrm{a}}$ Missing data in one patient with $\mathrm{CC} \geq 1$

${ }^{\mathrm{b}}$ Unknown cases in one patient with $\mathrm{CC}=0$ and in one patient with $\mathrm{CC} \geq 1$

${ }^{\mathrm{c}}$ Missing data in one patient with $\mathrm{CC}=0$
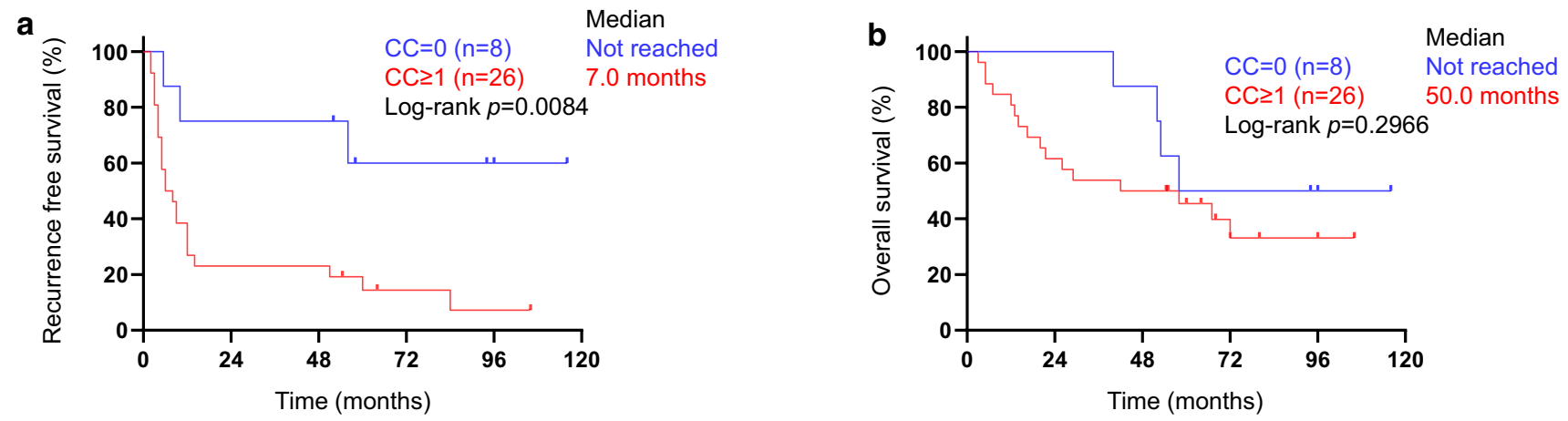

Number at risk:

\begin{tabular}{|c|c|c|c|c|c|c|}
\hline Time (month) & 0 & 24 & 48 & 72 & 96 & 120 \\
\hline $\mathrm{CC}=0$ & 8 & 7 & 7 & 4 & 2 & 1 \\
\hline $\mathrm{CC} \geq 1$ & 26 & 7 & 7 & 3 & 2 & 1 \\
\hline
\end{tabular}

Number at risk:
\begin{tabular}{|c|c|c|c|c|c|c|}
\hline Time (month) & 0 & 24 & 48 & 72 & 96 & 120 \\
\hline $\mathrm{CC}=0$ & 8 & 8 & 7 & 5 & 2 & 1 \\
\hline $\mathrm{CC} \geq 1$ & 26 & 17 & 14 & 6 & 3 & 2 \\
\hline
\end{tabular}

Fig. 1 Kaplan-Meier curves of recurrence-free survival (RFS) (a) and overall survival (OS) (b) for patients with mono-CC $(\mathrm{CC}=0)(\mathrm{blue}$ line) and poly- $\mathrm{CC}(\mathrm{CC} \geq 1)$ (red line)

\section{Association of clonal composition status with somatic mutations}

Somatic non-synonymous mutations in tissue samples $(n=36)$ were analyzed using targeted deep sequencing. Figure 2 summarizes the pathogenic variants with a FATHMM score $\geq 0.7$ among the somatic variants. Pathogenic mutations of TP53 $(9 / 36,25.0 \%)$ and $C T N N B 1(5 / 36,13.9 \%)$ were frequently identified mutations seen in the 36 samples that were analyzed, as reported previously [21]. No significant association was seen between the presence of a pathogenic TP53 gene mutation and grouping in the mono- and poly- CC groups $(12.5 \%$ vs. $30.8 \%, p=0.4030)$. Pathogenic CTNNB1 mutations tended to occur frequently in the mono$\mathrm{CC}$ group, but the difference was not significant (37.5\% vs. $7.7 \%, p=0.0721)$. The tumor mutation burden (TMB) has 


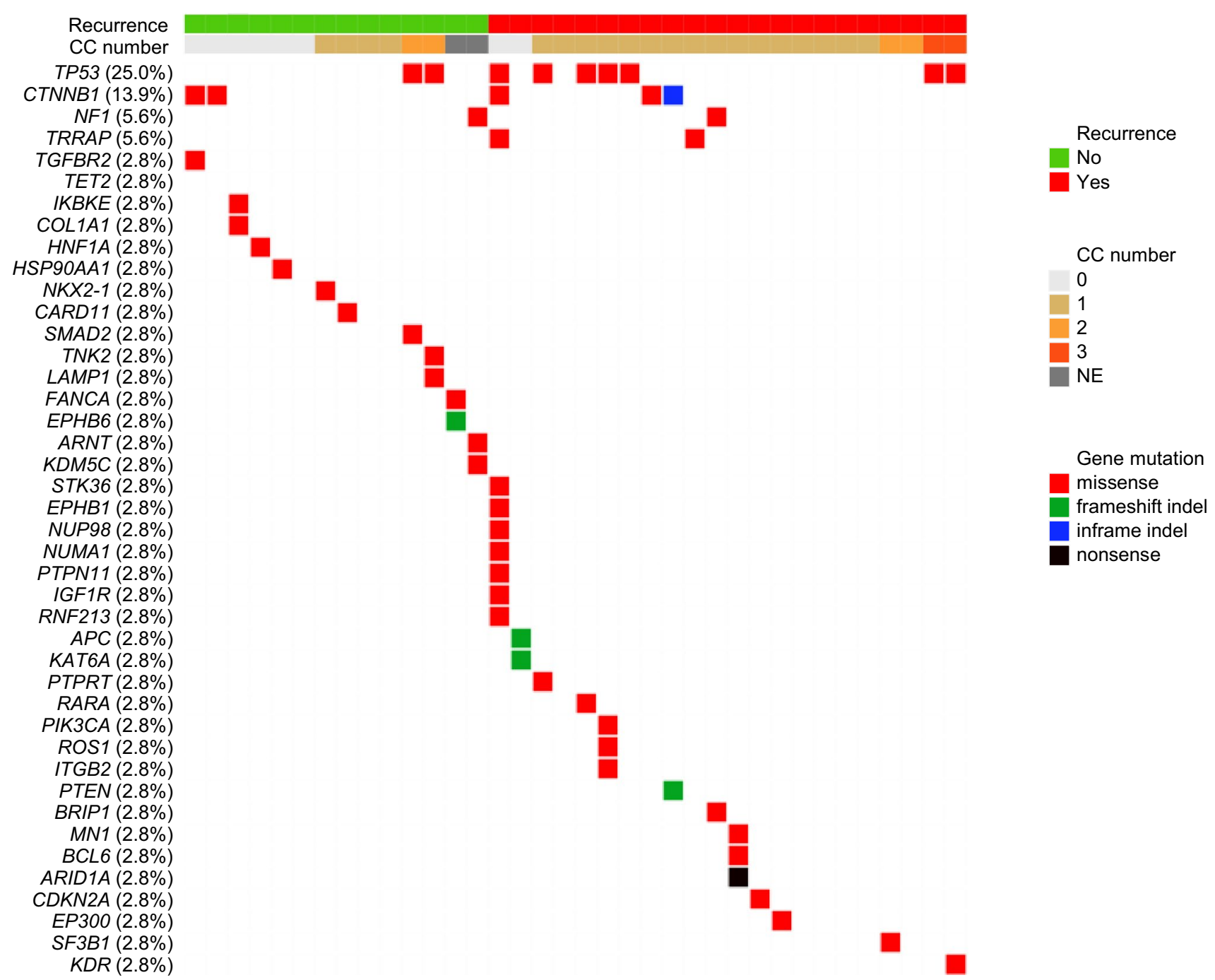

Fig. 2 Profiling of pathogenic mutations detected in HCC tissue samples. The different mutation types, CC numbers, and cases with recurrences are color-coded as indicated in the figure

been reported as the total number of non-synonymous variants per tumor genomic region [22]. The TMB level of HCC is relatively low, compared with other types of solid cancers [23]. The TMB score of the presently reported cohort was also not high (median TMB =6.7), and no difference in TMB was observed between the poly-CC (median 6.7; range 2.5-12.7) and the mono-CC (median 6.7; range 4.2-12.6) groups.

\section{Gene expression profile and functional enrichment analysis}

Thus far, our findings have indicated that the biological behavior of mono- and poly-CC tumors may differ. To investigate the biological functional differences between monoand poly-CC tumors, we performed gene-set enrichment and pathway analysis using gene expression data from the Ion
AmpliSeq Transcriptome Human Gene Expression assay. GSEA was performed for the enrichment of 50 hallmark gene sets from the MSigDB database. The enriched up- and down-regulated hallmarks with nominal $p<0.05$ and a false discovery rate (FDR) with $q<0.05$ are shown in Table 3 . The top five enriched gene set in the poly-CC group were related to E2F targets, the G2M checkpoint, Myc targets, the mitotic spindle, and DNA repair (Fig. 3a). On the other hand, the top five down-regulated signatures in the poly$\mathrm{CC}$ group were related Allograft rejection, IFN $\gamma$ response, Inflammatory response, TNF $\alpha$ signaling via NFKB, and IL6JAK-STAT3 signaling (Fig. 3b), indicating that the immune response is upregulated in mono-CC group.

The top 100 up-regulated and 100 down-regulated genes $(p<0.05)$ in poly-CC group are listed in Table S1. Among the 100 up-regulated genes in poly-CC, the number of genes corresponding to the E2F target, G2M checkpoint, 
Table 3 Gene set enrichment analysis (GSEA) results according to the MSigDB hallmark gene sets

\begin{tabular}{|c|c|c|c|c|}
\hline & MSigDB hallmark pathway & NES & NOM $p$ value & FDR $q$ value \\
\hline \multirow{12}{*}{$\begin{array}{l}\text { Upregulated } \\
\text { hallmark } \\
\text { signatures }\end{array}$} & $\mathrm{E} 2 \mathrm{~F}$ targets & 3.20 & 0 & 0 \\
\hline & G2M checkpoint & 3.12 & 0 & 0 \\
\hline & Myc targets v1 & 2.38 & 0 & 0 \\
\hline & Mitotic spindle & 2.31 & 0 & 0 \\
\hline & DNA repair & 2.02 & 0 & 0 \\
\hline & Myc targets v2 & 1.82 & 0 & 0.0003 \\
\hline & mTORC1 signaling & 1.81 & 0 & 0.0004 \\
\hline & Spermatogenesis & 1.69 & 0.0015 & 0.0020 \\
\hline & Oxidative phosphorylation & 1.58 & 0 & 0.0077 \\
\hline & Unfolded protein response & 1.58 & 0.0044 & 0.0069 \\
\hline & Protein secretion & 1.52 & 0.0104 & 0.0136 \\
\hline & Cholesterol homeostasis & 1.46 & 0.0162 & 0.0205 \\
\hline \multirow{18}{*}{$\begin{array}{l}\text { Downregulated } \\
\text { hallmark } \\
\text { signatures }\end{array}$} & Allograft rejection & -3.24 & 0 & 0 \\
\hline & Interferon gamma response & -3.13 & 0 & 0 \\
\hline & Inflammatory response & -2.97 & 0 & 0 \\
\hline & TNFa signaling via NFKB & -2.97 & 0 & 0 \\
\hline & IL6-JAK-STAT3 signaling & -2.89 & 0 & 0 \\
\hline & Epithelial mesenchymal transition & -2.75 & 0 & 0 \\
\hline & Interferon alpha response & -2.57 & 0 & 0 \\
\hline & Complement & -2.41 & 0 & 0 \\
\hline & IL2-STAT5 signaling & -2.26 & 0 & 0 \\
\hline & Coagulation & -2.15 & 0 & 0 \\
\hline & Apoptosis & -2.03 & 0 & 0 \\
\hline & Angiogenesis & -1.98 & 0.003 & 0 \\
\hline & KRAS signaling up & -1.94 & 0 & 0 \\
\hline & Hypoxia & -1.83 & 0 & 0.001 \\
\hline & UV response Dn & -1.66 & 0 & 0.003 \\
\hline & Xenobiotic metabolism & -1.66 & 0 & 0.003 \\
\hline & Myogenesis & -1.37 & 0.003 & 0.043 \\
\hline & Apical surface & -1.37 & 0.043 & 0.042 \\
\hline
\end{tabular}

NES normalized enrichment score, NOM nominal, FDR false discovery rate and mitotic spindle pathway were 19,19 , and 15 , respectively. The top five genes with a higher fold change (FC) of up-regulation in poly-CC related were $B I R C 5(\mathrm{FC}=4.85$, $p=0.0030), M Y B L 2(\mathrm{FC}=4.67, p=0.0144), C E N P F$ $(\mathrm{FC}=4.03, p=0.0007), D L G A P 5(\mathrm{FC}=4.03, p=0.0038)$, and NEK2 $(\mathrm{FC}=3.95, p=0.0010)$ genes. These genes are members of gene sets related to E2F targets, G2M checkpoint, and mitotic spindle pathways. In addition, gene expression level of MKI67, marker of proliferation Ki-67, was significantly higher in poly-CC compared with mono$\mathrm{CC}$ group $(\mathrm{FC}=2.75, p=0.0065)$. Among the 100 downregulated genes in poly-CC, the number of genes corresponding to the IFN $\gamma$ response, Inflammatory response, and IL6-JAK-STAT3 signaling were 3 (CCL2, KLRK1, and HLA-DMA), 4 (CSF3R, PCSK9, IFNGRI, and HMOXI), and $3(C S F 3 R, C C L 2$, and MARCO), respectively. The analysis of immune infiltrates affecting CC status showed a significant correlation between mono-CC and infiltration of macrophages (Fig. S4).

Previous reports revealed the association between the expression of OATP1B3, which is encoded by the gene $S L C O 1 B 3$, was strongly associated with $\mathrm{Wnt} / \beta$-catenin signaling [28]. SLCO1B3 gene expression was compared between mono-CC and poly-CC. Increased expression of SLCO1B3 was observed in mono-CC (mean $\pm \mathrm{SD}$, $2.98 \pm 2.08)$ compared with poly-CC (mean \pm SD, $1.69 \pm 2.24)(p=0.1173)$. Although not significantly different, expression of $S L C O 1 B 3$ tended to be higher in mono-CC. In addition, expression of $S L C O 1 B 3$ was higher in the CTNNB1 mutation positive cases (mean $\pm \mathrm{SD}$, $4.15 \pm 2.73$ ) than negative cases (mean $\pm \mathrm{SD}, 1.62 \pm 1.97$ ) $(p=0.0691)$. This suggests that mono-CC may be associated with $\mathrm{Wnt} / \beta$-catenin activated subtype $\mathrm{HCC}$. 


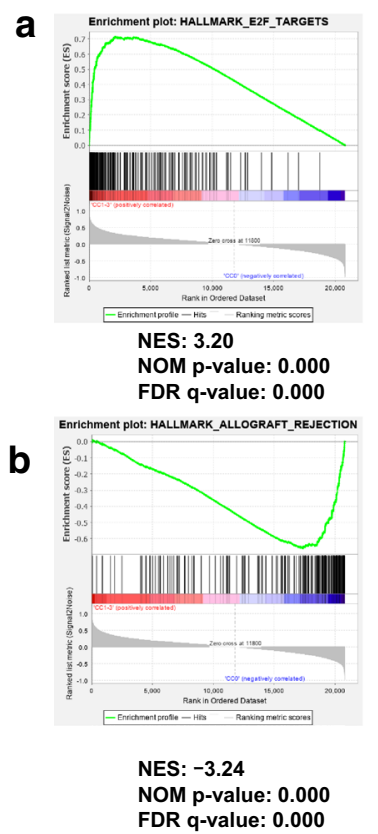

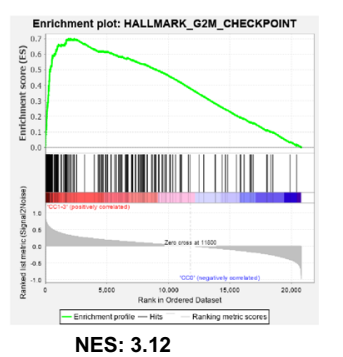

NOM p-value: 0.000

FDR q-value: $\mathbf{0 . 0 0 0}$

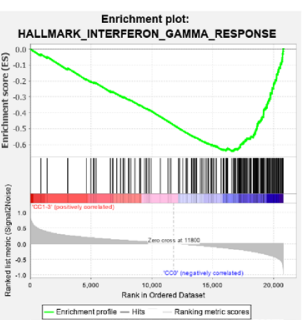

NES:-3.13

NOM p-value: 0.000

FDR q-value: $\mathbf{0 . 0 0 0}$

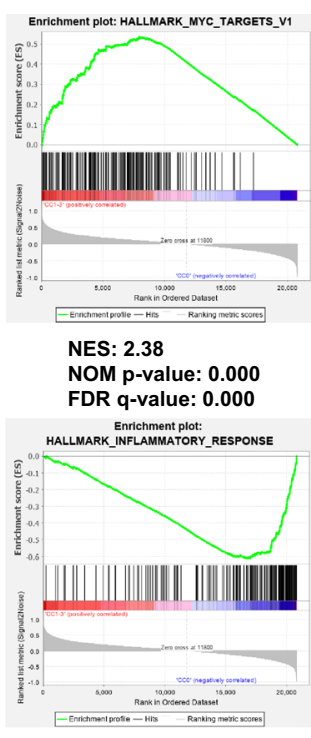

NES:-2.97 NOM p-value: 0.000 FDR q-value: 0.000
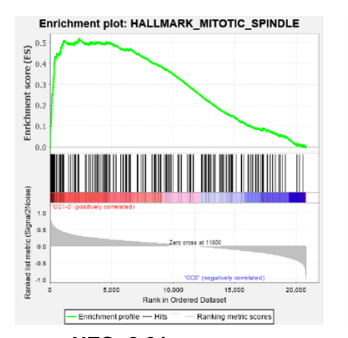

NES: 2.31

NOM p-value: 0.000

FDR q-value: 0.000

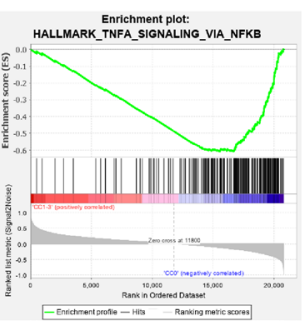

NES:-2.97

NOM p-value: 0.000 FDR q-value: 0.000

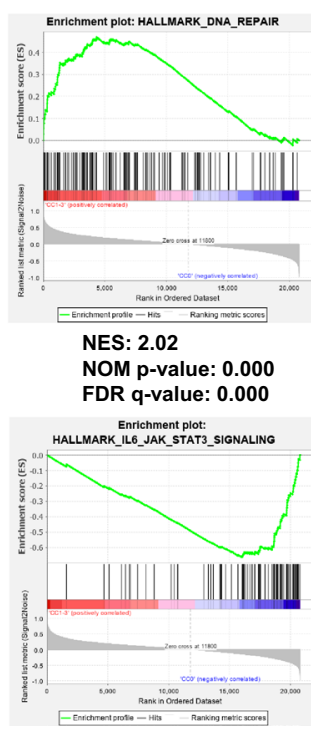

NES:-2.89

NOM p-value: 0.000 FDR q-value: 0.000

Fig. 3 Enrichment plots of gene expression signatures for top five upregulated hallmark signatures (a) and downregulated hallmark signatures (b) pathways in poly-CC tumors. NES, normalized enrichment score; NOM p value, Nominal p value; FDR q value: false discovery rate

To further explore this notion, we analyzed significant gene sets in the mono- and poly-CC tumors. A hierarchical clustering analysis of 500 differentially expressed genes revealed the enrichment of cluster I and cluster II genes in mono- and poly-CC tumors, respectively (Fig. 4a). Early recurrent patients exhibited the enrichment of cluster II genes. We conducted a gene ontology (GO) analysis using Metascape and found significant enrichment of the cellular pathways associated with the immune response and cytokine-related pathways in cluster I, which was enriched in mono-CC tumors (Fig. 4b). On the other hand, cell cycle-related pathways including those related to E2F targets, the G2M checkpoint, and DNA replication were enriched in cluster II, which was enriched in poly-CC tumors (Fig. 4c).

\section{Univariate and multivariate analyses for risk factors of early recurrence}

To identify the risk factors contributing to early postoperative recurrence of $\mathrm{HCC}$, univariate and multivariate analysis were performed (Table 4). Univariate analysis showed that NBNC HCC, large tumor size, and elevated PIVKA-II level were significantly correlated with early recurrence. In multivariate analysis, large tumor size was found to be a significant risk factor; $\mathrm{CC}$ status was not significant, but there was a trend toward a higher risk of early recurrence in poly-CC $(p=0.0846)$.

\section{Discussion}

Tumor heterogeneity is considered to be associated with a poor prognosis and outcome in cancer patients. In this study, we evaluated the CC number, a surrogate marker of intratumor heterogeneity, to investigate its clinical relevance in HCC. In general, HCC is reported to be highly heterogeneous [2-4]. In our sample set, the rate of homogeneous tumors with a CC number of 0 and the rate of heterogeneous tumors with a CC number of greater than 1 were $23.5 \%$ and $76.5 \%$, respectively, suggesting that HCC was often a heterogeneous tumor in our cohort. Heterogeneous tumors with poly-CC had a significantly higher rate of early recurrence. In addition, patients with poly-CC tumors were significantly associated with a poor postsurgical RFS. This finding suggests that $\mathrm{HCC}$ with high heterogeneity has a higher risk of recurrence after surgery. The $\mathrm{CC}$ number may be useful for predicting the risk of recurrence, but further studies are essential.

A gene mutation analysis was performed on the same sample set, and pathogenic mutations were selected using the FATHMM score. Therefore, the frequencies of TP53 and $C T N N B 1$ mutations were lower than previously reported [21]. The gene mutation analysis showed no association between the $\mathrm{CC}$ number and the gene mutations status, including the TMB. The calculation of $\mathrm{CC}$ number is based on the copy number loss, suggesting that the diversity of copy number variations is reflected by the $\mathrm{CC}$ number. In lung cancer, subclonal evolution based on copy number 


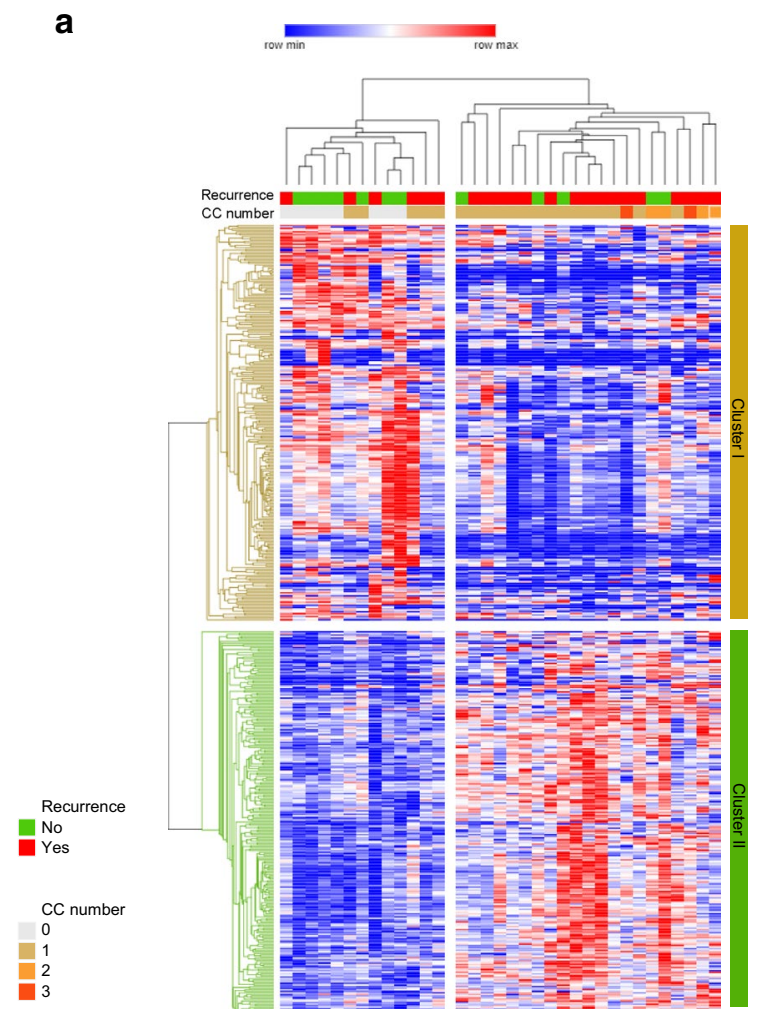

Fig. 4 Hierarchical clustering analysis of differentially expressed genes. a Hierarchical clustering analysis of 250 upregulated and 250 downregulated genes in poly-CC tumors compared with mono-CC tumors. Clustering was based on average linkage and the one minus Pearson correlation distance using Morpheus, and two major clusters (clusters I and II) were formed. b Plot shows the top biological path-

changes is reportedly associated with the RFS and OS to a greater degree than those based on single nucleotide mutations [24]. Evaluation of the CC number based on copy number loss may be more informative for predicting clinical outcome than the detection of single nucleotide variants in hepatocellular cancer.

In an enriched transcriptome analysis using a supervised approach, cell cycle-related pathways were among the top 5 upregulated pathways in poly-CC tumors. Oshi et al. showed that G2/M scoring is associated with cell proliferation-related factors and the breast cancer grade using an enrichment analysis based on a gene expression analysis of metastatic estrogen-receptor-positive breast cancer [25]. The pathways enriched in our cohort were related to the entire cell cycle progression in the G1, S and G2/M phases, suggesting active involvement in cell cycle progression and proliferation. The BIRC5, which was common to all three pathways and showed the highest difference, plays important rules in the E2F, G2M, and mitosis pathways, highlighting the importance of BIRC5 involvement in poly-CC tumor, particularly with regards to enhanced functions in cell cycle progression, division, and proliferation [21]. The b

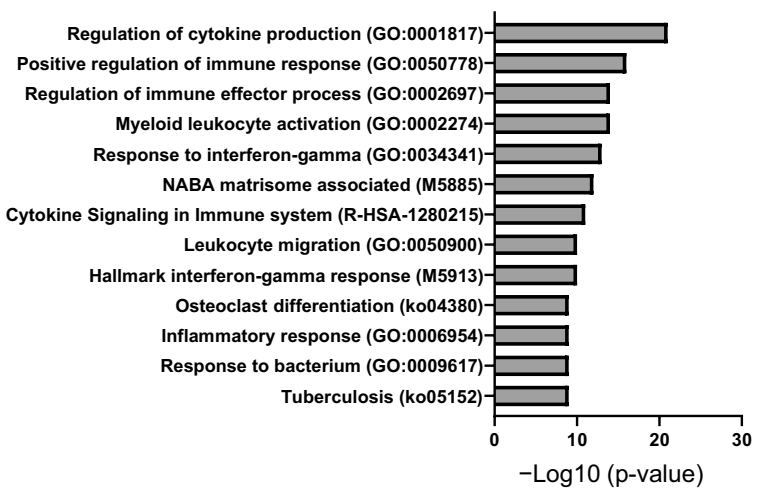

C

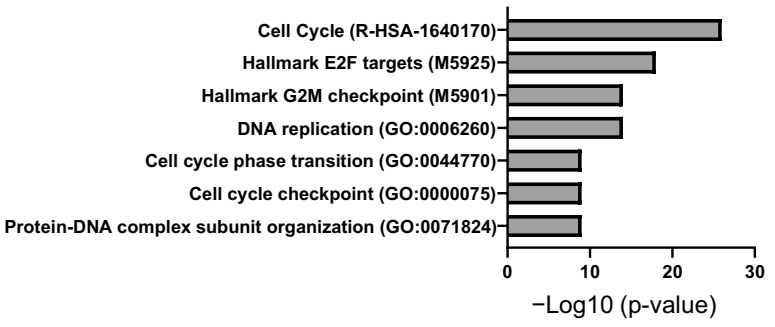

ways enriched in cluster I from the GO Biological Processes, Hallmark gene sets, and KEGG database using Metascape. c Plot shows the top biological pathways enriched in cluster II from the GO Biological Processes, Hallmark gene sets, and KEGG database using Metascape

other four genes have also been reported to be involved in proliferation in hepatocellular carcinoma [22-25], suggesting that these genes could be related to the increased proliferative phenotype of poly-CC. Recently, Llovet et al. [26]. reviewed the molecular classification of $\mathrm{HCC}$ that classifies HCC into "proliferation" and "non-proliferation" classes based on a previous transcriptome analysis [27]. The proliferation class is characterized by enriched pathways related to the cell cycle, TP53 mutation, and HBV-positive populations. Compared with the mono-CC group, the poly-CC group in our sample cohort tended to exhibit enriched E2F and other cell cycle pathways, a high TP53 mutation rate (8/26 [30.8\%] vs. $1 / 8$ [12.5\%]), and HBV positivity (5/26 [19.2\%] vs. $1 / 8$ [12.5\%]), although these differences were not significant because of the small sample size. Thus, polyCC tumors (cluster II) are consistent with these molecular and etiological characteristics of the "proliferative class." On the other hand, the non-proliferation class is associated with $\mathrm{HCV}$ and a molecular profile characterized by enhanced IL6-JAK-STAT signaling or CTNNB1 mutationmediated Wnt/ $\beta$-catenin signaling associated with inflamed pathogenicity [28-30]. Mono-CC tumors, compared with 
Table 4 Prognostic factors for early recurrence in surgical resected hepatocellular carcinoma

\begin{tabular}{|c|c|c|c|c|c|}
\hline Variable & Category & $\begin{array}{l}\text { Early recurrence } \\
(n=22)\end{array}$ & $\begin{array}{l}\text { No recurrence } \\
(n=14)\end{array}$ & $\begin{array}{l}\text { Univariate } \\
p\end{array}$ & $\begin{array}{l}\text { Multivariate } \\
p\end{array}$ \\
\hline Sex & Male/female & $13 / 9(59.1 / 40.9)$ & $9 / 5(64.3 / 35.7)$ & 1.0000 & - \\
\hline Age & $<70 / \geq 70$ & $11 / 11(50.0 / 50.0)$ & $4 / 10(28.6 / 71.4)$ & 0.3021 & - \\
\hline Liver function status & Child-Pugh A/B & $20 / 2(90.9 / 9.1)$ & $13 / 1(92.9 / 7.1)$ & 1.0000 & - \\
\hline HBV status & Positive/negative & $2 / 20(9.1 / 90.9)$ & $5 / 9(35.7 / 64.3)$ & 0.0842 & - \\
\hline HCV status & Positive/negative & $7 / 15(31.8 / 68.2)$ & $7 / 7(50.0 / 50.0)$ & 0.3142 & - \\
\hline NBNC & Yes/no & $13 / 9(59.1 / 40.9)$ & $2 / 12(14.3 / 85.7)$ & $0.0142 *$ & 0.4458 \\
\hline $\operatorname{ALT}(\mathrm{U} / \mathrm{l})$ & $<30 / \geq 30$ & $11 / 11(50.0 / 50.0)$ & $7 / 7(50.0 / 50.0)$ & 1.0000 & - \\
\hline Total bilirubin (mg/dl) & $<1 / \geq 1$ & $14 / 8(63.6 / 36.4)$ & $11 / 3(78.6 / 21.4)$ & 0.4672 & - \\
\hline Albumin (g/dl) & $<4 / \geq 4$ & $9 / 13(40.9 / 59.1)$ & $5 / 9(35.7 / 64.3)$ & 1.0000 & - \\
\hline ICGR15 (\%) & $<15 / \geq 15$ & $13 / 9(59.1 / 40.9)$ & $8 / 6(57.1 / 42.9)$ & 1.0000 & - \\
\hline $\operatorname{AFP}(\mathrm{ng} / \mathrm{ml})^{\mathrm{a}}$ & $<20 / \geq 20$ & $8 / 13(38.1 / 61.9)$ & $7 / 7(50.0 / 50.0)$ & 0.5108 & - \\
\hline PIVKA-II (mAU/ml) ${ }^{\mathrm{a}}$ & $<40 / \geq 40$ & 4/17 (19.0/81.0) & $10 / 4(71.4 / 28.6)$ & $0.0041 *$ & 0.2889 \\
\hline Tumor size $(\mathrm{cm})$ & $<4.0 / \geq 4.0$ & $6 / 16(27.3 / 72.7)$ & $12 / 2(85.7 / 14.3)$ & $0.0016^{*}$ & $0.0433 *$ \\
\hline Tumor number & Single/multiple & $19 / 3(86.4 / 13.6)$ & $10 / 4(71.4 / 28.6)$ & 0.3940 & - \\
\hline Microscopic vascular invasion & Positive/negative & 20/2 (90.9/9.1) & $10 / 4(71.4 / 28.6)$ & 0.1812 & - \\
\hline Histologic grade ${ }^{b}$ & Well/moderate, poor & $3 / 18(14.3 / 85.7)$ & $5 / 8(38.5 / 61.5)$ & 0.2106 & - \\
\hline Stage $^{c}$ & I, II/III, IVa & $4 / 18(18.2 / 81.8)$ & $5 / 8(38.5 / 61.5)$ & 0.2427 & - \\
\hline History of previous treatment & Present/absent & $3 / 19(13.6 / 86.4)$ & $4 / 10(28.6 / 71.4)$ & 0.3940 & - \\
\hline Clonal composition $^{\mathrm{d}}$ & Poly-CC/mono-CC & 20/2 (90.9/9.1) & $6 / 6(50.0 / 50.0)$ & $0.0127 *$ & 0.0846 \\
\hline
\end{tabular}

The asterisk indicates statistical significance

${ }^{a}$ Missing data in one patient with recurrence

${ }^{\mathrm{b}}$ Unknown cases in one patient with recurrence and in one patient with non-recurrence

${ }^{\mathrm{c}}$ Unknown cases in one patient with non-recurrence

${ }^{\mathrm{d}} \mathrm{CC}$ numbers could not be estimated in the two cases of patients without recurrence

poly-CC tumors, are characterized by non-HBV (7/8 [87.5\%] vs. $21 / 26[80.8 \%]), C T N N B 1$ mutation (3/8 [37.5\%] vs. $2 / 26$ [7.7\%]), and enriched cytokine-related pathways. The analysis of immune infiltrates affecting CC status showed a significant correlation between mono-CC and infiltration of macrophages (Fig. S4). This result might be linked to the enrichment of IFN $\gamma$ - and IL-6-mediated immune response pathway in mono-CC $[31,32]$. Thus, mono-CC tumors (cluster I) are consistent with these molecular and etiological characteristics of the "non-proliferative class".

Tumor heterogeneity of HCC may be different between tumor tissue of different etiology because of the disease features and the period of fibrogenesis as well the extent of chromosomal mutations and this phenomena is exemplified in this study which includes a heterogenous population HCC patients with different etiologies. The limitations of this study include its retrospective design and small sample size. Future studies examining larger sample sizes are needed to confirm the findings of this study and differential signatures of HCC with various etiologies. In the present study, pathway-related analysis was performed to characterize poly-CC tumors. However, the further analysis will be planned in the next study. In addition, isolation and transplantation of different poly-CC HCC cells in animals may elucidate copy number relevance in infiltration and proliferation rate.

In conclusion, HCCs with poly-CCs, a surrogate of heterogeneity based on copy number variation, are enriched in genes involved in the cell cycle pathway, have a proliferative phenotype, and have a higher risk of early postoperative recurrence.

Supplementary Information The online version contains supplementary material available at https://doi.org/10.1007/s12072-021-10278-4.

Acknowledgements We thank the participating patients and their families as well as all investigators and operations staff. The authors also thank Mr. Yoshihiro Mine (Center for Instrumental Analyses Central Research Facilities, Kindai University Faculty of Medicine) and Ms. Ayaka Kitano (Department of Genome Biology, Kindai University Faculty of Medicine) for technical assistance provided during the study.

Author contributions MK contributed substantially to the conception, design, and planning of the study, acquisition of data, analysis of the data, interpretation of the results, and drafting the manuscript; KS contributed substantially to the conception, design, and planning of the study, acquisition of data, analysis of the data, interpretation of the results, and drafting the manuscript; KN contributed substantially to the conception, design, or planning of the study, analysis of the data, 
interpretation of the results, and drafting the manuscript; HM, HK, $\mathrm{KM}, \mathrm{MAV}$, and MS contributed substantially to the acquisition of data, and interpretation of the results. All authors contributed substantially to critically reviewing or revising the manuscript for important intellectual content, and approved the final manuscript.

Funding This work was supported in part by a Grant-in Aid for Scientific Research (C) from the Japan Society for the Promotion of Science Grant Numbers JP19K07722 (KS).

Data Availability Yes. Data generated or analyzed during this study are available from the corresponding author on reasonable request.

\section{Declarations}

Conflict of interest Kazuko Sakai reports personal fees from AstraZeneca, Bio-Rad Laboratories, Chugai Pharmaceutical, Roche Diagnostics, Hitachi, outside the submitted work. Kazuto Nishio reports grants and personal fees from Eli Lilly, Nippon Boehringer Ingelheim, grants from Ignyta, Korea Otsuka Pharmaceutical, Thoracic Oncology Research Group, North East Japan Study Group, and personal fees from Chugai Pharmaceutical, Eisai, Pfizer, Novartis Pharma, MSD, Ono Pharmaceutical, Bristol-Myers Squibb, SymBio Pharmaceuticals, Life Technologies Japan, Solasia Pharma, Yakult Honsha, Roche Diagnostics, AstraZeneca, Otsuka Pharmaceutical, Sanofi, Guardant Health, Amgen, outside the submitted work. Marco A. De Velasco has received financial and material research support from AstraZeneca, Janssen and Taiho Pharmaceutical outside the submitted work. Masaki Kaibori, Hideyuki Matsushima, Hisashi Kosaka, Kosuke Matsui, and Mitsugu Sekimoto declare no conflicts of interest.

Animal research (ethics) Not applicable.

Plant reproducibility Not applicable.

Clinical trials registration Not applicable.

Consent to participate This study was conducted in compliance with the Helsinki Declaration and the ethical guidelines for medical and health research involving human subjects in Japan. This study was approved by the institutional ethics review boards of Kansai Medical University Hospital (\#1429) and Kindai University, Faculty of Medicine (27-033).

Consent to publish (ethics) Yes.

Open Access This article is licensed under a Creative Commons Attribution 4.0 International License, which permits use, sharing, adaptation, distribution and reproduction in any medium or format, as long as you give appropriate credit to the original author(s) and the source, provide a link to the Creative Commons licence, and indicate if changes were made. The images or other third party material in this article are included in the article's Creative Commons licence, unless indicated otherwise in a credit line to the material. If material is not included in the article's Creative Commons licence and your intended use is not permitted by statutory regulation or exceeds the permitted use, you will need to obtain permission directly from the copyright holder. To view a copy of this licence, visit http://creativecommons.org/licenses/by/4.0/.

\section{References}

1. Bray F, Ferlay J, Soerjomataram I, Siegel RL, Torre LA, Jemal A. Global cancer statistics 2018: GLOBOCAN estimates of incidence and mortality worldwide for 36 cancers in 185 countries. CA Cancer J Clin. 2018;68(6):394-424

2. Chan LK, Tsui YM, Ho DW, Ng IO. Cellular heterogeneity and plasticity in liver cancer. Semin Cancer Biol. 2021;. https://doi. org/10.1016/j.semcancer.2021.02.015.

3. Shimada S, Mogushi K, Akiyama Y, Furuyama T, Watanabe S, Ogura T, et al. Comprehensive molecular and immunological characterization of hepatocellular carcinoma. EBioMedicine. 2019;40:457-470

4. Wu Y, Liu Z, Xu X. Molecular subtyping of hepatocellular carcinoma: A step toward precision medicine. Cancer Commun (Lond). 2020;40(12):681-693

5. Carter NP. Methods and strategies for analyzing copy number variation using DNA microarrays. Nat Genet. 2007;39(7 Suppl):S16-21

6. Davies JJ, Wilson IM, Lam WL. Array CGH technologies and their applications to cancer genomes. Chromosome Res. 2005;13(3):237-248

7. Foster JM, Oumie A, Togneri FS, Vasques FR, Hau D, Taylor $\mathrm{M}$, et al. Cross-laboratory validation of the OncoScan(R) FFPE Assay, a multiplex tool for whole genome tumour profiling. BMC Med Genom. 2015;8:85

8. Sakai K, Ukita M, Schmidt J, Wu L, De Velasco MA, Roter A, et al. Clonal composition of human ovarian cancer based on copy number analysis reveals a reciprocal relation with oncogenic mutation status. Cancer Lett. 2017;405:22-28

9. Miller CA, White BS, Dees ND, Griffith M, Welch JS, Griffith OL, et al. SciClone: inferring clonal architecture and tracking the spatial and temporal patterns of tumor evolution. PLoS Comput Biol. 2014;10(8):e1003665

10. Kudo M, Kitano M, Sakurai T, Nishida N. General rules for the clinical and pathological study of primary liver cancer, nationwide follow-up survey and clinical practice guidelines: the outstanding Achievements of the Liver Cancer Study Group of Japan. Dig Dis. 2015;33(6):765-770

11. Yu B, Liang H, Ye Q, Wang Y. Establishment of a genomicclinicopathologic nomogram for predicting early recurrence of hepatocellular carcinoma after R0 resection. J Gastrointest Surg. 2021;25(1):112-124

12. Zhang XP, Chen ZH, Zhou TF, Li LQ, Chen MS, Wen TF, et al. A nomogram to predict early postoperative recurrence of hepatocellular carcinoma with portal vein tumour thrombus after R0 liver resection: a large-scale, multicenter study. Eur J Surg Oncol. 2019;45(9):1644-1651

13. Sakai K, Tsuboi M, Kenmotsu H, Yamanaka T, Takahashi T, Goto $\mathrm{K}$, et al. Tumor mutation burden as a biomarker for lung cancer patients treated with pemetrexed and cisplatin (the JIPANG-TR). Cancer Sci. 2021;112(1):388-396

14. Shihab HA, Rogers MF, Gough J, Mort M, Cooper DN, Day IN, et al. An integrative approach to predicting the functional effects of non-coding and coding sequence variation. Bioinformatics. 2015;31(10):1536-1543

15. Mootha VK, Lindgren CM, Eriksson KF, Subramanian A, Sihag $\mathrm{S}$, Lehar J, et al. PGC-1alpha-responsive genes involved in oxidative phosphorylation are coordinately downregulated in human diabetes. Nat Genet. 2003;34(3):267-273

16. Subramanian A, Tamayo P, Mootha VK, Mukherjee S, Ebert BL, Gillette MA, et al. Gene set enrichment analysis: a knowledgebased approach for interpreting genome-wide expression profiles. Proc Natl Acad Sci USA. 2005;102(43):15545-15550 
17. Zhou Y, Zhou B, Pache L, Chang M, Khodabakhshi AH, Tanaseichuk $\mathrm{O}$, et al. Metascape provides a biologist-oriented resource for the analysis of systems-level datasets. Nat Commun. 2019;10(1):1523

18. Li B, Severson E, Pignon JC, Zhao H, Li T, Novak J, et al. Comprehensive analyses of tumor immunity: implications for cancer immunotherapy. Genome Biol. 2016;17(1):174

19. Li T, Fan J, Wang B, Traugh N, Chen Q, Liu JS, et al. TIMER: a web server for comprehensive analysis of tumor-infiltrating immune cells. Cancer Res. 2017;77(21):e108-e110

20. Li T, Fu J, Zeng Z, Cohen D, Li J, Chen Q, et al. TIMER2.0 for analysis of tumor-infiltrating immune cells. Nucleic Acids Res. 2020;48(W1):W509-W514

21. Zucman-Rossi J, Villanueva A, Nault JC, Llovet JM. Genetic landscape and biomarkers of hepatocellular carcinoma. Gastroenterology. 2015;149(5):1226-1239 e1224

22. Samstein RM, Lee CH, Shoushtari AN, Hellmann MD, Shen $\mathrm{R}$, Janjigian YY, et al. Tumor mutational load predicts survival after immunotherapy across multiple cancer types. Nat Genet. 2019;51(2):202-206

23. Fessas P, Spina P, Boldorini RL, Pirisi M, Minisini R, Mauri FA, et al. Phenotypic characteristics of the tumour microenvironment in primary and secondary hepatocellular carcinoma. Cancers (Basel). 2021;13(9):2137

24. Jamal-Hanjani M, Wilson GA, McGranahan N, Birkbak NJ, Watkins TBK, Veeriah S, et al. Tracking the evolution of non-smallcell lung cancer. N Engl J Med. 2017;376(22):2109-2121

25. Oshi M, Takahashi H, Tokumaru Y, Yan L, Rashid OM, Matsuyama R, et al. G2M cell cycle pathway score as a prognostic biomarker of metastasis in estrogen receptor (ER)-positive breast cancer. Int J Mol Sci. 2020;21(8):2921
26. Llovet JM, Kelley RK, Villanueva A, Singal AG, Pikarsky E, Roayaie S, et al. Hepatocellular carcinoma. Nat Rev Dis Primers. 2021;7(1):6

27. Hoshida Y, Nijman SM, Kobayashi M, Chan JA, Brunet JP, Chiang DY, et al. Integrative transcriptome analysis reveals common molecular subclasses of human hepatocellular carcinoma. Cancer Res. 2009;69(18):7385-7392

28. Hoshida Y, Villanueva A, Kobayashi M, Peix J, Chiang DY, Camargo A, et al. Gene expression in fixed tissues and outcome in hepatocellular carcinoma. N Engl J Med. 2008;359(19):1995-2004

29. Hou J, Zhang H, Sun B, Karin M. The immunobiology of hepatocellular carcinoma in humans and mice: basic concepts and therapeutic implications. J Hepatol. 2020;72(1):167-182

30. Lachenmayer A, Alsinet C, Savic R, Cabellos L, Toffanin S, Hoshida Y, et al. Wnt-pathway activation in two molecular classes of hepatocellular carcinoma and experimental modulation by sorafenib. Clin Cancer Res. 2012;18(18):4997-5007

31. Muller E, Christopoulos PF, Halder S, Lunde A, Beraki K, Speth $\mathrm{M}$, et al. Toll-like receptor ligands and interferon-gamma synergize for induction of antitumor M1 macrophages. Front Immunol. 2017;8:1383

32. Wan S, Zhao E, Kryczek I, Vatan L, Sadovskaya A, Ludema G, et al. Tumor-associated macrophages produce interleukin 6 and signal via STAT3 to promote expansion of human hepatocellular carcinoma stem cells. Gastroenterology. 2014;147(6):1393-1404

Publisher's Note Springer Nature remains neutral with regard to jurisdictional claims in published maps and institutional affiliations. 